\title{
RUÍDO E EQUILÍBRIO: APLICAÇÃO DA POSTUROGRAFIA DINÂMICA EM INDÚSTRIA GRÁFICA
}

\section{Noise and Balance: the dynamic posturography in a printing industry}

\author{
Clarissa Stefani Teixeira ${ }^{(1)}$, Daiane Körbes ${ }^{(2)}$, Angela Garcia Rossi ${ }^{(3)}$
}

\section{RESUMO}

Objetivo: avaliar o equilíbrio corporal de indivíduos expostos a ruído ocupacional em uma indústria gráfica. Trata-se de um estudo descritivo, retrospectivo. Métodos: a medição objetiva do equilíbrio corporal foi realizada pelo método de Posturografia Dinâmica, utilizando-se o protocolo de Testes de Integração Sensorial. Foram avaliadas as respostas de 34 indivíduos do sexo masculino, divididos em dois grupos, sendo 16 trabalhadores expostos ao ruído com idade de 45,81 $\pm 7,38$ anos e 16 indivíduos sem exposição ao ruído ocupacional com idade de 41,31 $\pm 5,58$ anos. O tempo médio de exposição foi de $20,00 \pm 8,46$ anos. Os resultados foram comparados utilizando-se o teste $t$, considerandose como diferença estatística significante o valor de $p$ menor ou igual a 0,05. Resultados: todas as análises mostraram diferenças estatisticamente significantes entre os grupos, com os indivíduos expostos ao ruído ocupacional apresentando valores rebaixados de equilíbrio corporal em relação aos não expostos. Conclusão: a exposição ao ruído ocupacional está associada a um pior desempenho na avaliação do equilíbrio, em todas as condições testadas pela Posturografia Dinâmica.

DESCRITORES: Ruído Ocupacional; Doenças Profissionais; Equilíbrio Postural

\section{INTRODUÇÃO}

Uma das tarefas mais importantes do sistema de controle postural humano é a do equilíbrio do corpo sobre a base de apoio fornecida pelos pés. O sistema vestibular é uma das ferramentas mais importantes do sistema nervoso no controle da postura, atuando como um sensor de gravidade e fornecendo ao sistema nervoso central (SNC) informações sobre a posição e o movimento da cabeça e a direção da gravidade. O SNC, por sua vez, combina essas informações com as fornecidas por outros sistemas sensoriais e constrói uma

(1) Educadora Física; Universidade Federal de Santa Catarina, UFSC, Florianópolis, SC, Brasil; Mestre em Distúrbios da Comunicação Humana pela Universidade Federal de Santa Maria; Doutoranda em Engenharia de Produção na Universidade Federal de Santa Catarina.

(2) Fonoaudióloga; Hospital Bruno Born, Lajeado, RS, Brasil; Mestre em Distúrbios da Comunicação Humana pela Universidade Federal de Santa Maria.

(3) Fonoaudióloga; Professora Adjunta do Curso de Fonoaudiologia do Departamento de Fonoaudiologia da Universidade Federal de Santa Maria, UFSM, Santa Maria, RS, Brasil; Doutora em Ciências dos Distúrbios da Comunicação Humana pela Universidade Federal de São Paulo.

Conflito de interesses: inexistente imagem da posição e do movimento do corpo todo e do ambiente que o cerca ${ }^{1}$. O equilíbrio corporal é fundamental no relacionamento espacial do organismo com o ambiente. Três sistemas relacionamse entre si e com SNC, para a manutenção do equilíbrio: a visão, a sensibilidade proprioceptiva e 0 aparelho vestibular ${ }^{2-5}$. A correta postura do indivíduo será determinada pela interação harmoniosa desses três sistemas, em nível cerebral, juntamente com as memórias de experiências prévias ${ }^{6}$.

Como diversas estruturas estão envolvidas no processo de equilíbrio, a perda da estabilidade corporal, muitas vezes, pode estar relacionada a alterações no aparelho vestibular e/ou auditivo, ou ainda, depender de alterações à distância, em outros órgãos, sistemas ou mesmo forças externas 4,7. As manifestações dos distúrbios vestibulares incluem: desequilíbrio, desvios na marcha, instabilidade no andar, sensação de flutuação, sensação rotatória e quedas, podendo ocasionar ainda problemas psicossociais ${ }^{8}$ e prejuízos no trabalho ${ }^{9}$.

Dentre as principais etiologias das desordens vestibulares podem ser citadas as de origem vascular, cervical, metabólica, tóxica, tumoral, degenerativa, infecciosa, trauma crânico ou espinhal, barotrauma e trauma acústico. Os níveis de pressão 
sonora em ambiente ocupacional, com o passar dos anos, foram sendo intensificados e suas consequências potencializadas na medida em que as indústrias sentiram a necessidade de desenvolver e aperfeiçoar seus mecanismos de produção para auxiliar no trabalho humano. Dessa maneira, surgiram cada vez mais fatores físicos e ambientais prejudiciais à saúde auditiva, tais como ruído, temperatura, vibração, radiação e pressão em níveis elevados que, isolados ou em conjunto, causam danos ao trabalhador ${ }^{10}$.

Ao se correlacionar o ruído com sintomas vestibulares foi verificado que durante a exposição ao ruído ou mesmo após, muitos pacientes apresentam alterações tipicamente vestibulares, descritas como vertigens, que podem ou não ser acompanhadas de náuseas, vômitos, suores frios, dificuldades no equilíbrio e na marcha, nistagmos, desmaios e dilatação das pupilas ${ }^{11}$. O estímulo auditivo, antes de chegar ao córtex cerebral, passa por inúmeras estações subcorticais, principalmente pelas funções vegetativas, o que explica a presença dos efeitos não-auditivos induzidos pelo ruído ${ }^{12}$. Porém, indivíduos expostos a ruído ocupacional podem, muitas vezes, não se queixar de tonturas e vertigens provavelmente em decorrência da grande capacidade do aparelho vestibular em se adaptar ao dano gradativo imposto pelos sons de alta intensidade. Isto significa que alterações importantes podem ocorrer nas estruturas labirínticas sem a existência de muitas queixas por parte dos indivíduos. Por esta razão, o uso de protetores auriculares é importante não somente para proteger a audição, mas também para a preservação da função vestibular ${ }^{13-16}$.

Os autores ${ }^{17-20}$ indicam a necessidade permanente da adoção de medidas preventivas em relação à exposição ao ruído, tanto coletivas quanto individuais. Mesmo que atualmente existam programas de conservação auditiva, nota-se um elevado número de pessoas com diversas alterações provocadas pela exposição ao ruído. Alguns estudos foram encontrados relacionando as alterações otoneurológicas com a exposição ao ruído ocupacional 21-24. Entretanto, especificamente, estudos cujo foco esteja voltado às investigações das alterações nos mecanismos de manutenção do equilíbrio corporal possivelmente provocadas por elevados níveis de pressão sonora ainda são escassos na literatura. Logo, por este fato e pelos riscos à saúde do trabalhador que o ruído pode provocar, afetando adversamente o seu bem-estar, este estudo objetivou verificar a manutenção do equilíbrio corporal em diferentes condições sensoriais de indivíduos expostos ao ruído gerado em ambiente ocupacional.

\section{MÉTODOS}

Este estudo caracteriza-se como um estudo exploratório-descritivo, retrospectivo, de corte transversal, no qual será realizado um levantamento de informações ainda pouco investigadas em uma determinada população ${ }^{25}$.

Fizeram parte deste estudo 16 trabalhadores (GT) de uma gráfica da região sul do Brasil, do gênero masculino, com média de idade de $45,81 \pm 7,38$ anos, sem problemas relacionados ao sistema músculo-esquelético, hipertensão arterial e diabetes. Com relação ao sistema auditivo, dos 16 trabalhadores, $50 \%$ deles apresentavam limiares auditivos normais e 50\% apresentavam limiares auditivos rebaixados em frequências agudas. Todos os participantes do GT estavam expostos ao ruído por 8 horas diárias. Não foi exigido repouso auditivo para a realização da avaliação do equilíbrio corporal. O grupo controle (GC) foi constituído por 16 indivíduos que não estavam expostos ao ruído ocupacional, com média de idade de 41,31 \pm 5,58 anos, com as mesmas restrições do grupo de trabalhadores expostos ao ruído, porém sem problemas relacionados aos sistemas auditivo e vestibular. Todos os procedimentos foram explicados para ambos os grupos e aqueles que aceitaram participar do estudo assinaram um Termo de Consentimento Livre e Esclarecido, conforme resolução 196/1996 do Conselho Nacional da Saúde, do Ministério da Saúde.

Todos os funcionários preencheram, individualmente, um questionário elaborado pelos pesquisadores (Figura 1), com perguntas gerais e específicas relativas à exposição ao ruído, a fim de caracterizar o grupo do estudo, sendo garantido total sigilo da confidencialidade dos sujeitos.

Para a avaliação do equilíbrio corporal e suas relações com o sistema visual, proprioceptivo e vestibular, foi utilizada a posturografia dinâmica Foam-laser Dynamic Posturography (FLP) ${ }^{26}$. A escolha deste instrumento de coleta deu-se pelo mesmo testar, dentre as possibilidades de avaliação, a organização sensorial. A organização ou integração sensorial analisa a contribuição relativa dos receptores somatossensoriais, visuais e vestibulares na estabilidade global do indivíduo indicando qual dos sistemas utilizados para a manutenção do equilíbrio é o responsável pela instabilidade (padrões de distribuição sensorial) ${ }^{6}$, assim como sua capacidade de manter o equilíbrio com informações sensoriais erradas (visão preferencial) ${ }^{27,28}$.

A posturografia dinâmica expõe 0 indivíduo a seis condições distintas (I, II, III, IV, V, VI), na qual se anulam aferências sensoriais para analisar e comparar uma condição com a outra, compondo o 
DADOS DE IDENTIFICAÇÃO:

Nome:

D.N.:

Sexo:

Data:

Idade:

\section{DADOS GERAIS:}

1. Há quanto tempo trabalha exposto ao ruído?

Que tipo de ruído?

2. Qual o tempo de exposição diária?

3. Trabalha ou já trabalhou exposto a produtos químicos/tóxicos? ( ) Sim ( ) Não Qual(is)?

4. Você usa algum tipo de protetor auditivo? ( ) Sim ( ) Não

Se sim, qual o tipo: ( ) plug - dentro do ouvido ( ) concha - por cima da orelha

( ) outro:

Se não, explique por que?

5. Se faz uso de protetor, há quanto tempo?

6. Você freqüenta ambientes ruidosos além do local de trabalho? ( ) Sim ( ) Não Quais: ( ) bares ( ) boates ( ) academias ( ) pratica tiros ( ) corridas de moto ( ) outros:

7. Você sente dificuldade para ouvir? ( ) Sim ( ) Não

Qual orelha: ( ) direita ( ) esquerda ( ) ambas

Há quanto tempo?

8. Você tem dificuldade de compreender a fala? ( ) Sim ( ) Não

Com presença de ruído de fundo: ( ) Sim ( ) Não

Ao telefone: ( ) Sim ( ) Não

9. Sente zumbido (barulho) nos ouvidos? ( ) Sim ( ) Não

Qual orelha: ( ) direita ( ) esquerda ( ) ambas

Há quanto tempo?

Que tipo?

Prejudica a concentração no trabalho? ( ) Sim ( ) Não

Prejudica o sono? ( ) Sim ( ) Não

10. Você sente desconforto ao ouvir sons do meio ambiente, como o telefone, campainha, porta abrindo ou fechando, carro, ventilador? ( ) Sim ( ) Não

11. Já teve ou tem infecções no ouvido? ( ) Sim ( ) Não

12. Já teve ou tem dores no ouvido? ( ) Sim ( ) Não

13. Você já teve outros sintomas físicos devido à exposição prolongada ao ruído? Assinale:
( ) insônia
( ) dores de cabeça
( ) irritabilidade
( ) estresse
( ) falta de memória
( ) isolamento social
( ) depressão
( ) tremor das mãos
( ) falta de atenção
( ) enjôos
( ) ansiedade

( ) alteração da freqüência cardíaca

( ) outros:

14. Com que freqüência sente esses sintomas?
( ) sempre
( ) às vezes
( ) raramente
( ) nunca

15. Você tem:

Diabetes? ( ) Sim ( ) Não

Hipertireoidismo? ( ) Sim ( ) Não

16. Faz uso de medicação? ( ) Sim ( ) Não

Pressão alta? ( ) Sim ( ) Não

Hipotireoidismo? ( ) Sim ( ) Não

Qual(is)?

17. Você sente tonturas? ( ) Sim ( ) Não

Como é a tontura: ( ) objetos giram

( ) você gira

( ) oscilações

( ) instabilidade

( ) afundamento no solo

18. Há quanto tempo?

( ) outro: 
19. Qual a freqüência das crises de tontura?
( ) diariamente
( ) de 3 a 5 x/semana
( ) 1 ou $2 \times /$ semana
( ) a cada duas semanas ( ) a cada mês
( ) outro:

20. Quanto tempo dura cada crise?

21. Algum dos sintomas seguintes acompanha a tontura? Assinale:
( ) desmaio
( ) náusea
( ) vômito
( ) palidez
( ) suor frio nas mãos
( ) dor de cabeça
( ) zumbido
( ) queda
( ) visão embaralhada
( ) pressão no ouvido
( ) alteração da audição
( ) outro:

22. Algum dos fatores desencadeia a tontura? Assinale:
( ) girar a cabeça
( ) levantar-se rápido
( ) movimentar-se rápido
( ) olhar para os lados
( ) expor-se ao ruído
( ) outro:

23. Ingere bebida alcoólica? ( ) Sim ( ) Não

Com que freqüência?

Qual é a mais ingerida?

Qual a quantidade (em copos)?

24. Fuma? ( ) Sim ( ) Não

Quantos cigarros por dia?

\section{DADOS ADICIONAIS:}

1. Você já recebeu informações sobre perda de audição? ( ) Sim ( ) Não

2. Você considera o ruído nocivo para sua audição? ( ) Sim ( ) Não

3. Você acha que o ruído pode piorar uma perda auditiva já existente? ( ) Sim ( ) Não

4. A perda auditiva tem cura? ( ) Sim ( ) Não

5. Você já recebeu orientações sobre os cuidados com a audição? ( ) Sim ( ) Não

6. Qual a sua opinião sobre os protetores auditivos:
( ) oferece proteção suficiente
( ) prejudica a comunicação
( ) não oferece proteção suficiente
( ) não sabe a respeito
( ) desconfortável
( ) outro:

7. Qual profissional você procuraria se percebesse alguma alteração na audição:
( ) clínico geral
( ) fonoaudiólogo
( ) médico otorrinolaringologista
( ) outro:

Figura 1 - Questionário

Teste de Organização Sensorial (TOS). Para a avaliação por meio da FLP, cada indivíduo foi posicionado dentro de uma cabine de $1 \mathrm{~m}^{2}$, com altura de $2 \mathrm{~m}$, confeccionada com suporte de ferro desmontável, envolta por um tecido de algodão que cerca o indivíduo, estampado com listras horizontais claras e escuras, de $10 \mathrm{~cm}$ cada uma. A cabine é um sistema mecânico simples e move-se $20^{\circ}$ manualmente para frente e para trás durante o TOS III e VI. Uma caneta laser é fixada com a ponta estando verticalmente para cima através de um cinto confeccionado com espumas, cujas extremidades são adaptáveis à cintura de cada indivíduo. O laser é então projetado em um papel milimetrado de $50 \mathrm{~cm}$ x $50 \mathrm{~cm}$, fixado acima do corpo do indivíduo por um suporte de ferro. Para a manipulação do sistema somatossensorial, Castagno ${ }^{26}$ propõe a utilização de uma almofada de $10 \mathrm{~cm}$ de espessura entre os pés do indivíduo e o solo. Logo, o TOS I, II e III são executados sem a utilização desta almofada e os testes IV, V e VI com a utilização da mesma. Todas as condições são realizadas em posição ortostática, com os pés descalços ${ }^{27,28}$ e encontram-se relatadas a seguir:

- TOS I: a visão deve estar presente, com os olhos fixos na horizontal. A plataforma de apoio sob os pés é fixa, sem a utilização da almofada. A cabine visual deve permanecer imóvel durante 20 segundos. Neste teste foram avaliados os sistemas visual, proprioceptivo e vestibular.

- TOS II: a visão deve estar ausente, portanto, o indivíduo realiza o teste com os olhos fechados e, consequentemente, a cabine visual não possui movimento. A plataforma de apoio também se encontra imóvel, sem a utilização de 
almofada entre os pés e o solo. Este teste avalia os sistemas proprioceptivo e vestibular.

- TOS III: a visão deve estar presente e a referência (cabine visual) oscilante, sendo 10 segundos inclinada manualmente para frente e 10 segundos inclinada para retornar à posição inicial. $A$ plataforma de apoio ainda é fixa, ou seja, sem utilização da almofada. Esta condição avalia o sistema proprioceptivo, vestibular e, sobretudo o visual.

- TOS IV: engloba a visão normal, fixa na horizontal e a cabine visual, mais uma vez, imóvel durante 20 segundos. O suporte de apoio deve existir através da almofada entre os pés do indivíduo e o solo. O TOS IV avalia principalmente 0 sistema proprioceptivo.

- TOS V: a visão deve estar ausente (os olhos fechados), e a plataforma de apoio oscilante. Este teste avalia os sistemas proprioceptivo e vestibular, em condições de sobrecarga, devido à eliminação da aferência visual e a movimentação da plataforma.

- TOS VI: Este teste requer a visão normal com referência (cabine visual) e suporte de apoio oscilante. A avaliação é referente aos sistemas proprioceptivo, visual e vestibular.

A meta em cada uma destas condições de teste é a manutenção do equilíbrio estático. Em todas as condições testadas, o indivíduo é orientado a permanecer o mais imóvel possível na plataforma, mesmo diante das oscilações. Com base nos dados encontrados durante os deslocamentos, através da amplitude ântero-posterior, são calculadas as médias para cada um dos TOS. Depois destes cálculos, análises das funções somatossensorial e visual, preferência visual e índice de equilíbrio podem ser calculados através das médias das tentativas, inserindo as seguintes fórmulas:

- Função somatossensorial: TOS II/TOS I

- Função visual: TOS IV/TOS I

- Função vestibular: TOS V/TOS I

- Índice de equilíbrio: (TOS III + TOS VI)/(TOS II + TOS IV)

A pesquisa foi aprovada pelo Comitê de Ética e Pesquisa com Seres Humanos da Universidade Federal de Santa Maria e registrado sob o protocolo 23081.003726/2006-41.

Os dados referentes ao equilíbrio corporal foram processados em programa Excel. Para a análise dos dados utilizou-se estatística descritiva através da média e do desvio padrão. Primeiramente, para o tratamento estatístico, foi aplicado o teste de normalidade Shapiro-Wilk. Como os dados apresentaram distribuição normal, foi aplicado para a comparação entre os grupos de estudo e controle o teste $t$ de Student para dados independentes. O nível de significância adotado para todos os testes foi de $5 \%$.

\section{RESULTADOS}

Com base na análise dos questionários, observou-se que o tempo de exposição mínimo e máximo ao ruído para o grupo dos trabalhadores foi de 1 ano e 6 meses e 35 anos, respectivamente, com a média de exposição de $20,00 \pm 8,46$ anos. Quanto ao uso de protetor auricular, nenhum indivíduo referiu usá-lo de maneira regular.

Quanto aos achados obtidos com a aplicação da FLP, a Tabela 1 ilustra os valores da média e o desvio padrão durante a realização das 6 condições do TOS e sua média, para cada um dos grupos avaliados

Tabela 1 - Média e o desvio padrão, durante a realização das 6 condições de Organização Sensorial e sua média nos grupos controle (GC) e grupo dos trabalhadores expostos ao ruído (GT)

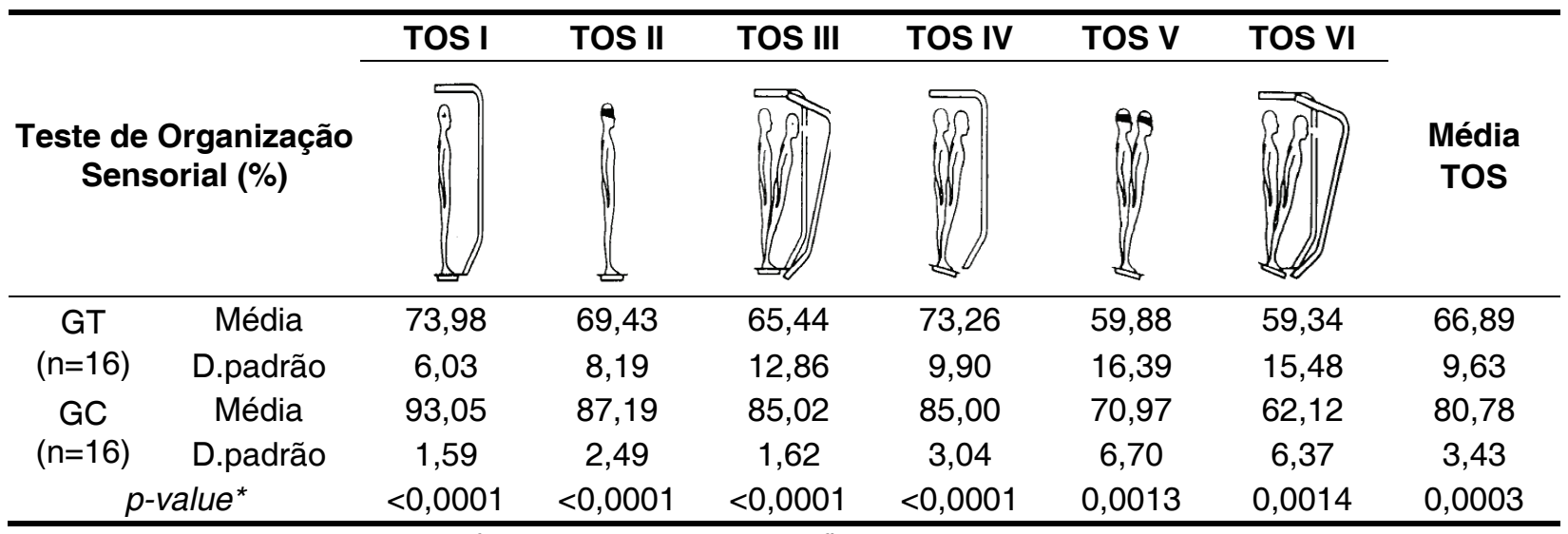

* Teste $t$ : valor menor que 0,05 indica diferença estatisticamente significante 
Tabela 2 - Média e o desvio padrão, da função somatosensorial, visual e vestibular e índice de equilíbrio dos dois grupos do estudo

\begin{tabular}{|c|c|c|c|c|c|}
\hline \multirow{2}{*}{$\begin{array}{c}\text { Análise sensorial } \\
(\%)\end{array}$} & \multicolumn{2}{|c|}{ GC $(n=16)$} & \multicolumn{2}{|c|}{ GT $(n=16)$} & \multirow{2}{*}{$p$-value } \\
\hline & Média & D. padrão & Média & D. padrão & \\
\hline & 94,14 & 10,96 & 93,71 & 2,50 & $<0,0001$ \\
\hline \multirow{3}{*}{$\begin{array}{l}\text { \&IS VIS } \\
\text { VEST }\end{array}$} & 98,82 & 9,71 & 90,68 & 2,38 & $<0,0001$ \\
\hline & 80,35 & 20,93 & 76,15 & 6,37 & $<0,0001$ \\
\hline & 96,11 & 16,80 & 93,10 & 3,28 & $<0,0001$ \\
\hline
\end{tabular}

* Teste $t$ : valor menor que 0,05 indica diferença estatisticamente significante

A Tabela 2 ilustra os valores da média e do desvio padrão da função somatosensorial, visual, preferência visual e índice de equilíbrio dos trabaIhadores da gráfica e do grupo controle. Os valores encontrados relacionados ao teste de organização sensorial (TOS de I a VI) e a analise sensorial (somatossensorial, visual, vestibular e preferência visual) mostraram diferenças estatisticamente significantes quando os diferentes grupos foram comparados. Além disso, os resultados apresentados pelo grupo controle foram superiores (melhores) ao grupo de trabalhadores expostos ao ruído.

\section{DISCUSSÃO}

A posturografia dinâmica vem sendo utilizada na prática clínica como um complemento à bateria de testes para diagnóstico otoneurológico e se mostra uma ferramenta útil na pesquisa de alterações do equilíbrio ${ }^{27,29-32}$ não diagnosticadas pelos testes básicos da função vestibular. Além disso, a posturografia é considerada por alguns autores como um método mais sensível do que os testes vestibulares convencionais na detecção de alterações vestibulares causadas pela exposição ao ruído ${ }^{33}$.

$\mathrm{Na}$ posturografia, as principais condições que avaliam a função vestibular são aquelas que permitem a oscilação da plataforma, neste caso, a utilização da almofada posicionada entre o solo e os pés do indivíduo, obtida nas condições TOS IV, V e VI. Nessas situações a oscilação fisiológica do centro de gravidade do corpo desloca a superfície de apoio com o balanço corporal e obriga o indivíduo a utilizar a informação vestibular para corrigir seu ângulo de tornozelo e não cair. Nessa condição, o sistema vestibular é a aferência cuja precisão da informação tem o maior impacto na estabilização corporal. Isso ocorre porque as informações vestibulares são as mais fiéis diante de um ambiente que oferece aferências conflitantes quando comparadas às informações visuais e proprioceptivas ${ }^{27,28,34}$. No presente estudo, além das diferenças encontradas nos testes IV, V e VI, houve alterações no equilíbrio corporal também durante a execução dos outros testes, demonstrando a influência do ruído no equilíbrio corporal em todas as condições avaliadas.

Mesmo que os referenciais teóricos relacionados a estudos com equilíbrio corporal e ruído tenham sido uma limitação do estudo, as citações encontradas inferem que há associação entre a exposição ao ruído em ambiente de trabalho e o desequilíbrio. De acordo com a literatura, além dos conhecidos efeitos prejudiciais do ruído para a audição, este interfere negativamente no equilíbrio e pode desencadear alterações neurovegetativas e psicossomáticas, como redução da capacidade de concentração, diminuição da eficiência no trabaIho, interferência na comunicação, perturbação do sono, influência sobre o sistema endócrino, distúrbios gástricos, cefaleias, nervosismo, cansaço, problemas de memória, irritação, tontura e vertigem ${ }^{35}$.

Em estudos experimentais realizados com cobaias expostas a sons intensos foi encontrada relação direta entre a exposição ao ruído e presença de alterações histológicas no labirinto vestibular. Autores encontraram como ocorrência mais comum a ruptura de uma fina lâmina de células que separa o utrículo e os canais semicirculares do sáculo e do resto do vestíbulo. A parede do sáculo estava rompida em aproximadamente 1/4 dos espécimes, e colapsada em cerca de $1 / 3$ deles ${ }^{21}$. Em outra pesquisa, realizada com ruídos de impulso - de 90 a 300 
tiros de rifle - foi verificado que as cristas ampulares dos animais foram mais intensamente danificadas, sendo observadas ainda alterações nas máculas do utrículo e sáculo ${ }^{23}$.

Em uma pesquisa com pombos submetidos à estimulação sonora prolongada, no período de uma hora, a $100 \mathrm{~dB}$ em uma das orelhas, foi observado trauma no labirinto vestibular comparável ao trauma produzido na cóclea. Especificamente os efeitos dos sons traumáticos sobre a função dos canais semicirculares também foram estudados. A estimulação sonora aplicada reduziu a sensibilidade do órgão sensorial dos canais semicirculares, indicando que, assim como a cóclea, o utrículo e o sáculo, os órgãos sensoriais dos canais semicirculares são susceptíveis a traumas sonoros, e que esta sensibilidade varia de animal para animal, visto que alguns mostraram alterações permanentes, outras alterações reversíveis ou ainda nenhuma alteração labiríntica ${ }^{22}$.

O ruído produzido por máquinas de indústria gráfica, nesta pesquisa, pode estar influenciando na manutenção do equilíbrio corporal em todos os sistemas relacionados à estabilidade. Resultados semelhantes, que confirmam a relação do ruído com alterações do equilíbrio também foram encontrados em outra pesquisa, que avaliou 15 trabaIhadores expostos ao ruído por meio da avaliação audiológica e da vectoeletronistagmografia computadorizada (VENG). A autora verificou que $40 \%$ dos indivíduos apresentaram alteração à VENG, todos com diagnóstico de síndrome vestibular periférica irritativa, concluindo que o ruído pode afetar adversamente os sistemas auditivo e vestibular, gerando ainda uma série de sintomas, potencializadas pela negligência ao uso do protetor auricular ${ }^{24}$. Em consonância com o estudo, a negligência ao uso dos equipamentos de proteção individual já foi evidenciada por pesquisa de Tipple et al ${ }^{36}$.

Quanto a estudos que utilizaram a posturografia que, da mesma forma, correlacionaram perdas auditivas, ruído e equilíbrio, autores utilizaram exames clínicos, neurológicos, audiológicos e medidas do equilíbrio corporal com plataforma estável, em 115 indivíduos normais (grupo controle) e em 60 pacientes que tinham sido expostos a ruídos intermitentes de alta energia por arma de fogo, apresentando variados graus de perda auditiva induzida por ruído (PAIR). Os sujeitos expostos ao ruído mostraram desequilíbrio corporal significantemente maior do que os controles, condizendo com os resultados aqui encontrados. Porém, segundo os mesmos autores, o desequilíbrio correlacionou-se muito pouco com os níveis de exposição a ruídos estimados, embora indivíduos com perdas auditivas mais intensas mostrassem maior desequilíbrio do que aqueles com perdas auditivas menos intensas. Tais resultados sugerem distúrbios subclínicos do sistema vestibular entre os indivíduos com perda auditiva induzida por ruído de impulso ${ }^{37}$.

Em uma análise de 3.000 casos de distúrbios vestibulares por intermédio da posturografia (EquiTest), pesquisadores definiram como perda vestibular os casos com alterações no TOS V e VI, associados à hipofunção do reflexo vestíbulo-ocular; e, como deficiência vestibular, os casos com TOS $\mathrm{V}$ e VI alterados, mas com ausência de anormalidade vestíbulo-ocular ou hiperfunção de reflexo vestíbulo-ocular. Além das alterações encontradas pelos autores nas condições específicas, os mesmos concluíram que a posturografia se mostrou um teste altamente sensitivo ${ }^{38}$.

Utilizando os mesmos grupos de estudo, diferentes autores ${ }^{33,37}$ desenvolveram um estudo, com posturografia, a fim de identificar a diferença entre a intensidade da PAIR. Com relação à perda auditiva a intensidade do ruído, o tempo de exposição e a idade parecem ser os principais fatores de risco para perda auditiva, independentemente da banda de frequência ${ }^{39-42}$ estatisticamente maiores desequilíbrios do que os de referência. Entre os que tinham PAIR mais intensa foi encontrado o maior desequilíbrio comparando com os que apresentaram menor PAIR. Os autores sugeriram que o mecanismo envolvido pode estar relacionado ao dano mecânico simultaneamente aos segmentos coclear e vestibular pelo ruído de impulso intenso ${ }^{33,37}$. Outra pesquisa foi desenvolvida para avaliar o efeito do ruído de impulso na estabilidade postural de 54 indivíduos do exército finlandês que sofriam de perda auditiva aguda causada por exposição a armas de fogo, num período máximo de cinco dias após a exposição. Os autores usaram como referência um grupo controle de 59 pessoas nãoexpostas a ruídos. Os diferentes sistemas receptores que controlam a postura como, por exemplo, os efeitos da visão, função de propriocepção, foram passo a passo excluídos ou alterados, deixando principalmente o controle postural vestibular intacto, para poderem avaliar exclusivamente a função dos órgãos otolíticos na orientação postural. Contrariamente aos resultados encontrados no presente estudo, os autores não observaram diferença entre os controles normais e os pacientes do exército expostos a ruídos em qualquer das condições de testes usadas, não havendo, portanto, correlação entre equilíbrio corporal e severidade de perda auditiva. Logo, os autores concluíram que o ruído de impulso não é causa de mudanças funcionais significantes no sistema vestibular ${ }^{42}$. 
De forma geral, pode-se inferir que as diferenças encontradas com a exposição ao ruído sugerem que a posturografia pode auxiliar na averiguação de alterações do equilíbrio corporal provocadas pela exposição em ambientes com níveis de pressão sonora elevados, assim como em outros casos.

\section{CONCLUSÃO}

Todos as condições relacionadas com o equilíbrio corporal mostraram-se estatisticamente diferentes para os grupos estudados, sendo que os indivíduos com exposição ao ruído ocupacional apresentaram valores mais baixos, ou seja, maiores desequilíbrios corporais quando comparados aos indivíduos sem exposição ao ruído.

Desta forma, sugere-se a continuidade de pesquisas que busquem a identificação precoce de alterações no equilíbrio corporal decorrentes do ruído, pois, mesmo que não seja possível acabar com essa problemática, pode-se procurar impedir o seu agravamento e as possíveis interferências nas atividades de vida diária e nos relacionamentos interpessoais decorrentes da exposição ao ruído ocupacional.

\begin{abstract}
Purpose: to assess the body balance of individuals exposed to occupational noise in a printing industry. This is a descriptive, retrospective study. Methods: the objective measurement of body balance was performed by the method of Dynamic Posturography, using the protocol of Sensorial Integration Tests. We evaluated the responses of 34 male subjects, divided into two groups, with 16 workers exposed to noise aged $45.81 \pm 7.38$ years and 16 subjects with no exposure to occupational noise aged $41.31 \pm 5.58$ years. The average exposure time was $20.00 \pm 8.0046$ years. The results were compared using the $t$ test, considering statistically significant differences as the $p$ value less than or equal to 0.05 . Results: all tests showed statistically significant differences among the groups, with individuals exposed to occupational noise showing demoted values of body balance in relation to nonexposed ones. Conclusion: the occupational noise exposure is associated with a worse performance in the assessment of balance in all conditions tested by Dynamic Posturography.
\end{abstract}

KEYWORDS: Occupational Noise; Occupational Diseases; Postural Balance

\section{REFERÊNCIAS}

1. Mazzucato A, Borges APO. Influência da reabilitação vestibular em indivíduos com desequilíbrio postural. Rev Neurocienc. 2009; 17(2):183-8.

2. Bankoff ADP, Bekedorf R. Bases neurofisiológicas do equilíbrio corporal. Efdeportes. [periódico on line] 2007 Mar; 11(106). [acesso em 19 Nov 2009] Disponível em: http://www.efdeportes.com/efd106/ bases-neurofisiologicas-do-equilibrio-corporal.htm

3. Zanardini FH, Zeigelboim BS, Jurkiewicz AL, Marques JM, Martins-Bassetto J. Reabilitação vestibular em idosos com tontura. Pró-Fono. 2007; 19(2):177-84.

4. Sturnieks DL, George R, Lord SR. Balance disorders in the elderly. Clin Neurophysiol. 2008; 38(6):467-78. http://dx.doi.org/10.1016/j. neurocli.2008.09.001
5. Patel M, Fransson PA, Lush D, Gomez S. The effect of foam surface properties on postural stability assessment while standing. Gait Post. 2008; 28(4):649-56. http://dx.doi.org/10.1016/j. gaitpost.2008.04.018

6. Mendonça AC, Rossi AG, Flores FT, Teixeira CS. Alterações do equilíbrio em indivíduos ex-usuários de álcool e drogas ilícitas. Acta ORL. 2006; 24(4):255-8.

7. Freitas Júnior PF, Barela JA. Alterações no funcionamento do sistema de controle postural de idosos. Uso da informação visual. Rev Port Cien Desp. 2005; 6(1):94-105.

8. Guida HL. Efeitos psicossociais da perda auditiva induzida pelo ruído em ex-funcionários da indústria. Acta ORL. 2007; 25(1):78-83.

9. Moretti MM, Prado IMM. Nível de pressão sonora produzido em experimentos sobre a velocidade do som e a sua ação sobre o organismo humano. Arq Mudi. 2007; 11(1):34-40. 
10. Medeiros LB. Ruído: efeitos extra-auditivos no corpo humano [monografia]. Porto Alegre (RS): CEFAC - Pós-Graduação em Saúde e Educação; 1999.

11. Gómez JG. Sordera por ruído. El traumatismo acústico y los accidentes auditivos en la industria. Bol Oficina Sanit Panam. 1983; 95(1):14-20.

12. Okamoto VA, Santos VP. Outros efeitos do ruído no organismo. In: Santos VP. Org. Ruído, riscos e prevenção. São Paulo: Hecitec; 1996. p.89-91.

13. Laitinen $\mathrm{H}$. Factors affecting the use of hearing protectors among classical music players. Noise Health. 2005; 7(26):21-9.

14. Mendes MH, Morata TC, Marques JM. Aceitação de protetores auditivos pelos componentes de banda instrumental e vocal. Rev Bras Otorrinolaringol. 2007; 73(6):785-92.

15. Milonski J, Olszewski J. [The evaluation of usefullnes of hearing protectors while exposure to impulse noise]. Otolaryngol Pol. 2007; 61(5):877-9.

16. Costa CB, Gama WU, Momensohn-Santos TM. Eficácia do protetor auditivo de inserção em Programa de Prevenção de Perdas Auditiva. Arq. Int. Otorrinolaringol. 2009; 13(3):281-6.

17. Gonçalves CGO. Análise do programa de apoio e reabilitação para trabalhadores portadores de PAIR em uma metalúrgica. Dist Comun. 2007; 19(1):103-16.

18. Lopes AC, Otubo KA, Basso TC, Marinelli ÉJI, Lauris JRP. Perda auditiva ocupacional: audiometria tonal $\mathrm{X}$ audiometria de altas frequências. Arq. Int. Otorrinolaringol. 2009; 13(3):293-9.

19. Lopes AC, Nelli MP, Lauris JRP, Amorim RB, Melo ADP. Condições de saúde auditiva no trabalho: investigação dos efeitos auditivos em trabalhadores expostos ao ruído ocupacional. Arq. Int. Otorrinolaringol. 2009; 13(1):49-54.

20. Ogido R, Costa EA, Machado HC. Prevalência de sintomas auditivos e vestibulares em trabalhadores expostos a ruído ocupacional. Rev Saúde Pública. 2009; 43(2):377-80.

21. Mangabeira-Albernaz PL, Covell WP, Eldredge $\mathrm{DH}$. Changes in the vestibular labyrinth with intense sound. Laryngoscope. 1959; 69(12):1478-93. http:// dx.doi.org/10.1288/00005537-195912000-00002

22. Van Eyck M. Sound-produced labyrinthine trauma. Arch Otolaryngol. 1974; 100(6):465-6.

23. Ylikoski J. Delayed endolymphatic hydrops syndrome after heavy exposure to impulse noise. Am J Otol. 1988; 9(4):282-5.

24. Körbes D. Avaliação do sistema vestibulococlear de trabalhadores de uma indústria gráfica de Santa Maria expostos a ruído ocupacional [monografia]. Santa Maria (RS): Universidade Federal de Santa Maria; 2007.
25. Thomas JR, Nelson JK. Métodos de pesquisa em atividade física. Porto Alegre: ArtMed; 2002.

26. Castagno LA. A new method for sensory organization tests: the foam-laser dynamic posturography. Rev Bras Otorrinolaringol. 1994; 60(4):287-96.

27. Loth EA, Rosi AG, Cappellesso PC, Ciena AP. Avaliação da influência do sistema vestibular no equilíbrio de adultos jovens através de posturografia dinâmica foam-laser e plataforma de força de Cascavel-PR. Semina Cienc Biol Saúde. 2008; 29(1): 57-64.

28. Teixeira CS. Hidroginástica na reabilitação vestibular de idosos com queixas de tontura [dissertação]. Santa Maria (RS): Universidade Federal de Santa Maria; 2008.

29. Bakker M, Allum JH, Visser JE, Grüneberg C, van de Warrenburg BP, Kremer BH, et al. Postural responses to multidirectional stance perturbations in cerebellar ataxia. Exp Neurol. 2006; 202(1):21-35.

30. Leitner C, Mair P, Paul B, Wick F, Mittermaier $\mathrm{C}$, Sycha $\mathrm{T}$, et al. Reliability of posturographic measurements in the assessment of impaired sensorimotor function in chronic low back pain. $J$ Electromyogr Kinesiol. 2009; 19(3):380-90.

31. Suarez $H$, Angeli $S$, Suarez A, Rosales $B$, Carrera X, Alonso R. Balance sensory organization in children with profound hearing loss and coclear implants. Int J Pediatr Otorhinolaryngol. 2007; 71(4):629-37.

32. Novalo ES, Pedalini MEB, Bittar RSM, Lorenzi MC, Bottino MA. Posturografia dinâmica computadorizada: avaliação quantitativa de pacientes com vestibulopatia tratados por meio de reabilitação vestibular. Arq. Int. Otorrinolaringol. 2008; 12(2):253-7.

33. Ylikoski J. Impulse noise induced damage in the vestibular end organs of the guinea pig. A light microscopic study. Acta Otolaryngol. 1987; 103(5-6):415-21.

34. Flores FT. Equilíbrio corporal de indivíduos com doença de Parkinson. [dissertação]. Santa Maria (RS): Universidade Federal de Santa Maria; 2009.

35. Aita ADC, Gonçalves AA. Avaliação do handicap auditivo em trabalhadores expostos a ruído. J Bras Fonoaudiol. 2001; 2(7):160-4.

36. Tipple AFV, Aguliari HT, Souza ACS, Pereira MS, Mendonça ACC, Silveira C. Equipamentos de proteção em centros de material e esterilização: disponibilidade, uso e fatores intervenientes à adesão. Cienc Cuid Saúde. 2007; 6(4):441-8.

37. Juntunen J, Matikainen E, Ylikoski J, Ylikoski $M$, Ojala M, Vaheri E. Postural body sway and exposure to high-energy impulse noise. Lancet. 1987; 2(8553):261-4. 
38. Hamid MA, Hughes GB, Kinney SE. Specificity and sensitivity of dynamic posturography: a retrospective analysis. Acta Otolaryngol. Suppl. 1991; 481:596-600.

39. Caldart AU, Adriano CF, Terruel I, Martins RF, Caldart AU, Mocellin M. Prevalência da perda auditiva induzida pelo ruído em trabalhadores de indústria têxtil. Arq. Int. Otorrinolaringol. 2006; 10(3):192-6.

40. Lopes G, Ruddo ICP, Fiorini AC. Estudo da audição e da qualidade de vida em motoristas de caminhão. Rev. CEFAC. 2007; 9(4):532-42. http:// dx.doi.org/10.1590/S1516-18462007000400014

41. Andára SB. Perfil audiológico de trabalhadores expostos ao ruído usuários da unidade de saúde do trabalhador do município de Gravataí. [monografia]. Porto Alegre (RS): Universidade Federal do Rio Grande do Sul; 2008.

42. Pyykkö I, Aalto H, Ylikoski J. Does impulse noise induce vestibular disturbances? Acta Otolaryngol. Suppl. 1988; 468:211-6.

DOI: 10.1590/S1516-18462010005000016

RECEBIDO EM: 28/09/2009

ACEITO EM: 04/12/2009

Endereço para correspondência:

Daiane Körbes

Rua Miguel Ruschel, 153

Estrela - RS

CEP: 95880-000

E-mail: daia_up@yahoo.com.br 